\title{
Effects of Cluster Sets and Rest-Redistribution on Mechanical Responses to Back Squats in Trained Men
}

\author{
by \\ James J. Tufano ${ }^{1,2}$, Jenny A. Conlon ${ }^{2}$, Sophia Nimphius ${ }^{2,3}$, Lee E. Brown ${ }^{4}$ \\ Alex Petkovic ${ }^{2}$, Justin Frick², G. Gregory Haff'
}

\begin{abstract}
Eight resistance-trained men completed three protocols separated by 48-96 hours. Each protocol included 36 repetitions with the same rest duration, but the frequency and length of rest periods differed. The cluster sets of four (CS4) protocol included $30 \mathrm{~s}$ of rest after the 4 th, 8th, 16th, 20th, 28th, and 32nd repetition in addition to $120 \mathrm{~s}$ of rest after the 12th and 24th repetition. For the other two protocols, the total $420 \mathrm{~s}$ rest time of CS4 was redistributed to include nine sets of four repetitions (RR4) with $52.5 \mathrm{~s}$ of rest after every four repetitions, or 36 sets of single repetitions (RR1) with $12 \mathrm{~s}$ of rest after every repetition. Mean (MF) and peak (PF) force, velocity (MV and PV), and power output $(M P$ and $P P)$ were measured during 36 repetitions and were collapsed into 12 repetitions for analysis. Repeated measures ANOVA 3 (protocol) $x 12$ (repetition) showed a protocol $x$ repetition interaction for $P F, M V, P V, M P$, and $P P$ (p-values from <0.001 to 0.012). No interaction or main effect was present for MF. During RR1, MV, PV, MP, and $P P$ were maintained, but decreased throughout every 4-repetition sequence during CS4 and RR4. During CS4 and $R R 4, P F$ was less following a rest period compared to subsequent repetitions, whereas $P F$ was maintained during RR1. These data indicate that rest redistribution results in similar average kinetics and kinematics, but if total rest time is redistributed to create shorter but more frequent sets, kinetics and kinematics may remain more constant.
\end{abstract}

Key words: athletic performance, muscle strength, power output, resistance training, velocity, weight lifting.

\section{Introduction}

Although sport-specific training is paramount for athletes of all sports, periodized resistance training helps foster optimal performance and reduce the risk of injury (Faigenbaum et al., 2009; Myer et al., 2005). To increase the effectiveness of resistance training, acute sessions should include systematic overload stimuli in order for the body to experience and adapt to increases in systemic stress (Selye, 1950). Oftentimes, this is done by increasing the external training load during resistance training (Fry, 2004). However, as training load increases, rest periods are generally modified in order to successfully complete a prescribed number of repetitions (de Souza et al., 2010; Medeiros et al., 2013). Therefore, the modification of inter-set and intra-set rest periods have received considerable attention within the scientific strength and conditioning literature.

Previous research has shown that cluster sets, which contain intra-set rest periods, maintain acute mechanical performance (i.e. force, movement velocity, and power output) better than traditional sets which contain no intra-set rest (Hardee et al., 2012; Tufano et al., 2016b). Since intra-set rest periods allow for the replenishment of immediate energy stores, the removal of metabolic byproducts from the

\footnotetext{
1 - Faculty of Physical Education and Sport, Charles University, Prague, Czech Republic.

2 - Centre for Exercise and Sports Science Research, Edith Cowan University, Joondalup, Australia.

3 - Hurley Surfing Australia High Performance Centre, Casuarina, Australia.

4 - Center for Sport Performance, California State University Fullerton, California, USA.
} 
muscle, and the maintenance of acute performance (Girman et al., 2014; Oliver et al., 2015), cluster sets have been used to perform high volumes of external work without resulting in greater acute neuromuscular fatigue (Joy et al., 2013; Oliver et al., 2015; Tufano et al., 2016b) in a variety of exercises and populations (Asadi and Ramirez-Campillo, 2016; Iglesias-Soler et al., 2013).

To create cluster sets, some researchers have added intra-set rest without changing the inter-set rest duration, increasing the total rest time (Haff et al., 2003; Hardee et al., 2012; Tufano, et al., 2016b); whereas, others have equated the total rest time between protocols by redistributing the total inter-set rest time throughout the protocol (Joy et al., 2013; Lawton et al., 2006; Oliver et al., 2015). These studies have generally implemented $30 \mathrm{~s}$ of inter-repetition rest (Haff et al., 2003; Hardee et al., 2012; Moir et al., 2013), with a range from 6 s (García-Ramos et al., 2015) to upwards of over $40 \mathrm{~s}$ (Iglesias-Soler et al., 2014). Some studies include a different number of repetitions for each participant, resulting in individualized rest redistribution that cannot directly be compared to other participants within the same study or of other studies. Most studies have compared a single traditional set protocol to a single cluster set protocol, and in the few studies that have compared cluster set protocols to each other (Hardee et al., 2012; Lawton et al., 2006; Moreno et al., 2014), comparisons were not made between basic cluster sets with additional intra-set rest periods and the "rest redistribution" technique. Hence, data comparing different cluster set structures (i.e. the addition of intra-set rest versus the redistribution of total rest time) are lacking. By examining such protocols, valuable information may be gathered regarding how the duration and frequency of rest periods influence neuromuscular fatigue during resistance training.

Therefore, the purpose of this study was to determine the effect of a basic cluster set inclusive of a standard inter-set rest period with the addition of intra-set rest and two different rest redistribution protocols with different rest period frequencies on kinetics and kinematics during back squats in trained men. Based on previous literature (Moreno et al., 2014), it was hypothesized that the protocol with the most frequent, but shortest, rest periods would result in greater movement velocities and power outputs compared to protocols with longer but less frequent rest periods when the total rest time was equated between protocols.

\section{Methods}

\section{Participants}

Eight resistance-trained males participated in this study $(25.2 \pm 4.1 \mathrm{y} ; 76.7 \pm 5.1$ $\mathrm{kg} ; 1.75 \pm 0.07 \mathrm{~m}$ ). All participants had at least six months of strength training experience using the back squat exercise and were able to perform a free weight back squat (top of the thighs at or below parallel) with at least $150 \%$ of their body mass with an average one-repetition maximum $(1 \mathrm{RM})$ of $135.0 \pm 16.8 \mathrm{~kg}$, equating to a $1 \mathrm{RM}$ to body mass ratio of $1.76 \pm 0.22$, with a peak knee flexion angle at the bottom of the squat of $129.5 \pm$ $11.5^{\circ}$. Participants were screened using medical history questionnaires and were excluded if they reported any recent musculoskeletal injuries. The Human Research Ethics committee at the Edith Cowan University approved all procedures and all participants gave written informed consent prior to participation.

\section{Procedures}

Participants reported to the laboratory to determine 1RM during a single session (Matuszak et al., 2003) followed by three randomized experimental sessions. Each session occurred at the same time each morning and was separated by $48-96 \mathrm{~h}$. Participants were instructed to avoid all other forms of exercise for $48 \mathrm{~h}$ leading up to data collection for the duration of the study and abstained from eating and drinking during the protocols. Each of the experimental sessions included 36 back squat repetitions using $75 \%$ of 1RM. The total assigned rest time was equal between protocols, but the distribution of rest varied. The cluster sets of four (CS4) protocol included $30 \mathrm{~s}$ of rest after the $4^{\text {th }}, 8^{\text {th }}, 16^{\text {th }}, 20^{\text {th }}, 28^{\text {th }}$, and $32^{\text {nd }}$ repetition in addition to $120 \mathrm{~s}$ of rest after the $12^{\text {th }}$ and $24^{\text {th }}$ repetition (Tufano et al., 2016a). For the other two protocols, the total rest time was redistributed to include nine sets of four repetitions (RR4) with $52.5 \mathrm{~s}$ of rest provided after every four repetitions, or 36 sets of single repetitions (RR1) with $12 \mathrm{~s}$ of rest provided after every repetition. Therefore, all three protocols included 36 repetitions at $75 \%$ of $1 \mathrm{RM}$ with $420 \mathrm{~s}$ of standing, unloaded rest (Figure 1). 
During each of the experimental sessions, participants were instructed to "squat all the way down" by lowering the barbell under control and to "explode out of the bottom" by performing each squat as quickly as possible during the concentric phase without jumping and without the bar leaving the shoulders (Cormie et al., 2007). Participant foot placement was kept constant for every repetition during every session using a horizontal-vertical grid marked on the force plate with individualized visual markings. Following a standard dynamic warm-up during each session which included stationary cycling, a dynamic warm-up and squats with progressively increasing loads, each protocol began when participants positioned themselves under the barbell at the beginning of a verbal five-second countdown. When the countdown reached zero, the participants un-racked the bar and stepped backwards onto the force plate to perform the desired number of consecutive repetitions according to the assigned protocol. After completing one (RR1) or four (CS4 and RR4) repetitions, the participants re-racked the bar in the squat rack and remained standing while unloaded during the rest period. The participants positioned themselves under the barbell when the next five-second countdown began and the process was repeated until the protocol was finished.

\section{Measures}

All squats were performed on a force plate to measure mean force (MF) and peak force $(\mathrm{PF})$ and two linear transducers were attached to each side of the barbell (four in total) originating from the top of the squat rack to calculate an overall vector of barbell movement and to obtain mean velocity (MV) and peak velocity (PV). External mechanical mean power (MP) and peak power (PP) of the system were calculated by direct measurement of ground reaction force and bar velocity. All kinematic and kinetic data were collected using methodology similar to previous research (Cormie et al., 2007) and all variables were collected during the concentric phase of each lift as previously defined. A customized LabVIEW program (National Instruments, Version 14.0, Austin, TX) was used to collect and manually analyze data received from the force plate (AMTI BP12001200; Watertown, MA) and four linear position transducers (Celesco PT5A-250;
Chatsworth, CA) via a BNC-2090 interface box with an analog-to-digital card (NI-6014; National Instruments, Austin TX, USA). All signals were sampled at $1000 \mathrm{~Hz}$ and filtered using a 4th orderlow pass Butterworth filter with a cut-off frequency of $50 \mathrm{~Hz}$ with retraction tension of the four linear transducers $(23.0 \mathrm{~N})$ accounted for in all calculations.

\section{Statistical Analysis}

The means and standard deviations for all 36 repetitions were averaged by collapsing across 12-repetition segments within each protocol (i.e. repetition $1=(1+13+25) / 3$; repetition $2=$ $(2+14+26) / 3$; etc.), similar to previous research that reduced data in order to compare different protocols during high-volume resistance-training sessions (Oliver et al., 2016). For each independent variable, a $3 \times 12$ (protocol $\times$ repetition) repeated measures ANOVA was used within SPSS version 22.0 (IBM, Armonk, NY). Similar to previous research (Joy et al., 2013), in the event of a significant protocol $x$ repetition interaction, Tukey's follow-up pairwise comparisons were compared in 4-repetition segments to maintain consistency between protocols, due to the design of RR4 and CS4 (i.e. repetitions 1-4 were compared independent of repetitions 5-12; repetitions 5-8 compared independent of repetitions 1-4 and 9-12; and repetitions 9-12 were compared independent of repetitions 1-8). Statistical significance was set at $p \leq 0.05$ for all tests.

\section{Results}

Means and standard deviations for all variables are shown in Table 1 . There were no significant between protocol differences for any variable when all 36 repetitions were averaged together. However, a protocol $\mathrm{x}$ repetition interaction was present for PF, MV, PV, MP, and PP (Table 1). Significant differences are indicated in Figure 2. There was neither an interaction nor a main effect for the protocol for MF.

\section{Discussion}

Basic cluster sets and rest redistribution protocols have been investigated independently within the scientific literature (Tufano et al., 2017), but have not been compared within the same study. Therefore, the purpose of this study was to determine the effect of a basic cluster set inclusive 
of a standard inter-set rest period with the addition of intra-set rest and two different rest redistribution protocols with different rest period frequencies on the kinetics and kinematics of back squats in trained men. The main finding of the present study was that when using the same load and number of repetitions, the mean acute kinetic and kinematic responses to free-weight back squats were similar regardless of how the rest periods were distributed within the session, but the patterns of each variable were different between protocols, with the exception of MF. Specifically, the first repetition following a rest period in RR4 and CS4 displayed less PF than the following three consecutive repetitions: a pattern that was not present in RR1. Additionally, MV, PV, MP, and PP all progressively decreased throughout every 4-repetition segment, which did not occur during RR1.

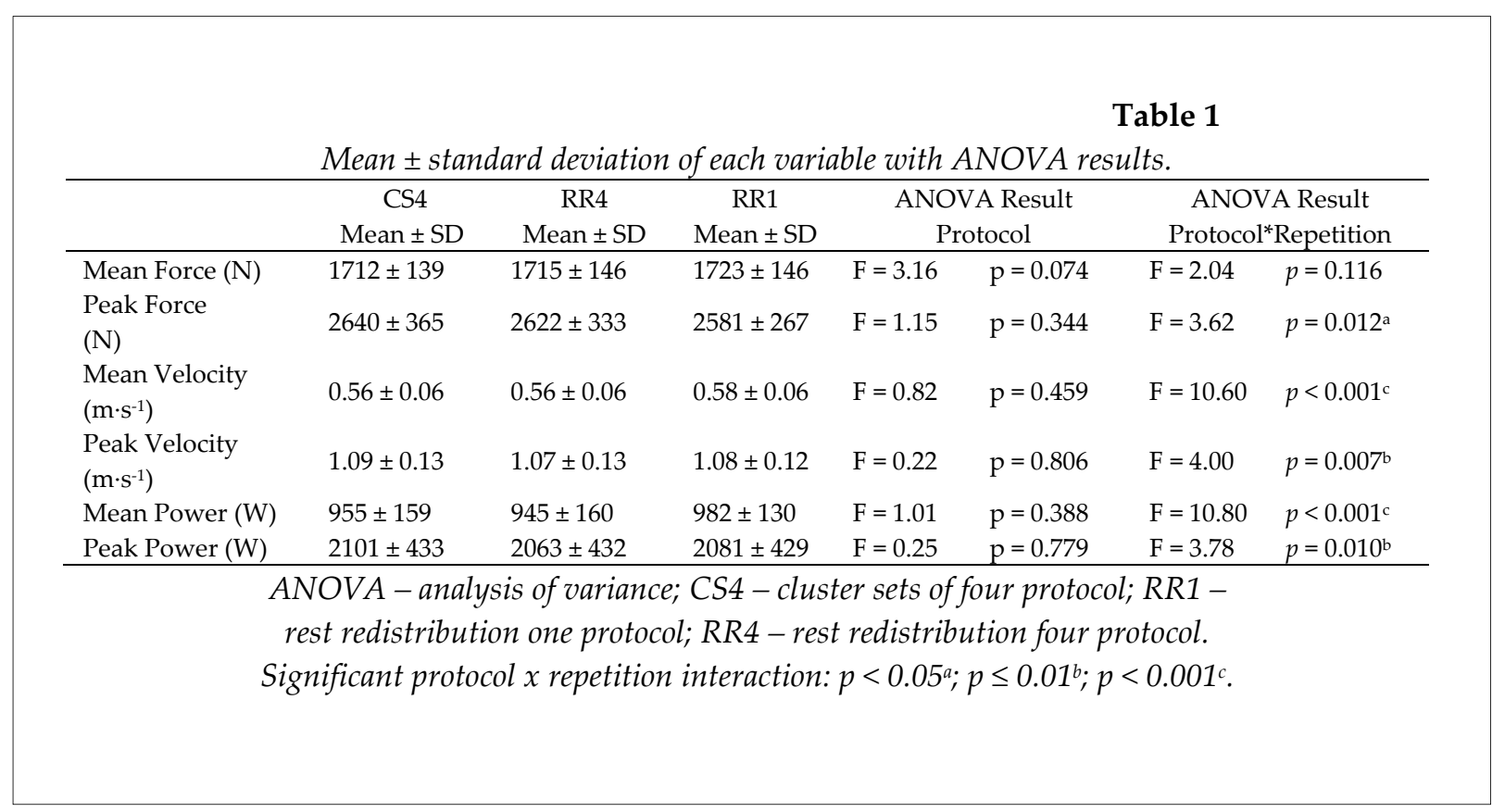

CS4

IIII_IIII_IIII__IIII_IIII_IIII___IIII_IIII_IIII

RR4

IIII----IIII----IIII----IIII----III|----IIII----IIII----|III|----IIII

Inter-Set Rest: 8 x $52.5 \mathrm{~s}=420 \mathrm{~s} \quad$ Intra-Set Rest: N/A Total Rest: $420 \mathrm{~s}$

RR1

I.I.I.I.I.I.I.I.I.I.I.I.I.I.I.I.I.I.I.I.I.I.I.I.I.I.I.I.I.I.I.I.I.I.I.I

Inter-Set Rest: $35 \times 12 \mathrm{~s}=420 \mathrm{~s}$

Intra-set Rest: N/A

Total Rest: $420 \mathrm{~s}$

Figure 1

Cluster set protocol with 420 s of total rest (CS4), redistributed to create

nine sets of four repetitions with $52.5 \mathrm{~s}$ of inter-set rest (RR4) and to

create thirty-six sets of one with 12 s of inter-repetition rest (RR1). 

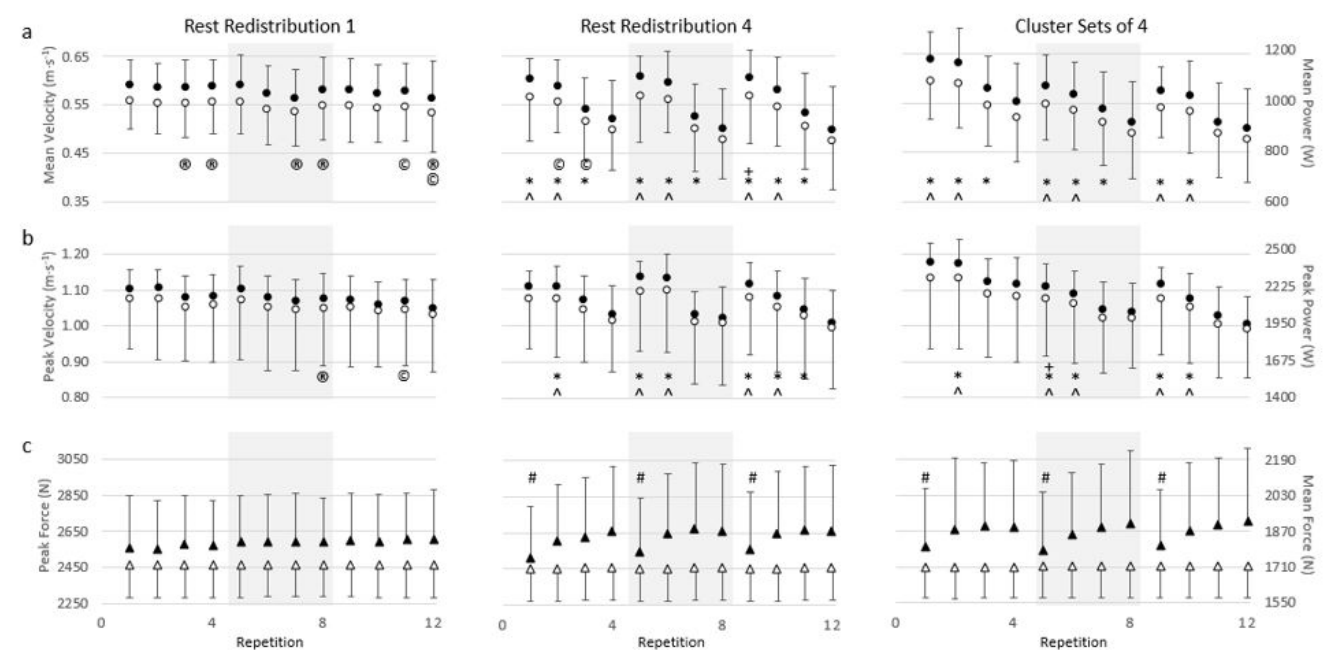

Figure 2(a)

Mean velocity and power output, 2(b) peak velocity and power output, and 2(c) peak force output collapsed into twelve-repetition segments for each protocol. Closed circles indicate velocity data on the primary vertical axis and open circles for power data on the secondary vertical axis. Closed triangles show peak force and open triangles show mean force.

Significantly greater than the $* 4^{\text {th }}, \wedge 3^{r d}$, and $+2^{\text {nd }}$ repetition of each segment; Significantly different from the same repetition of the CCS4 protocol and the ${ }^{\circledR} R R 4$ protocol; Peak force significantly less than the following three repetitions \#

Despite mean MV and PV of all 36 repetitions being statistically similar between protocols, there were different velocity and power output responses between the protocols (Figure 2a and $2 b$ ). Furthermore, MP and PP mirrored the $\mathrm{MV}$ and PV responses, supporting the hypothesis that movement velocity is largely responsible for the production of external power output (Oliver et al., 2016). In RR1, velocity and power output remained fairly steady; but, when four repetitions were performed in a row regardless of the protocol (RR4 and CS4), a decrease in velocity and power output occurred in each 4-repetition segment. Therefore, despite a lack of significant differences in the global kinematic responses, the patterns observed in the protocol $\mathrm{x}$ repetition interactions of the present study should be considered by strength and conditioning professionals as the data indicate that practical training implications may arise when prescribing resistance exercises if acute movement velocity is of interest.

In order to discuss the practical applications of such observations, the role of monitoring velocity during acute resistancetraining should be understood. To abide by the training principle of specificity, some athletes and coaches strive to acutely achieve maximal movement velocity and power output, hypothesizing that chronic exposure to such stimuli will result in positive training adaptations that translate into heightened performance. 
Therefore, some coaches and researchers implement "velocity-based training" protocols in which a minimum velocity threshold (i.e. $80 \%$ of the maximal attainable velocity for a given load) must be maintained to avoid overly fatiguing the neuromuscular system (Jovanović and Flanagan, 2014; Padulo et al., 2012; Pareja-Blanco et al., 2016). According to the recommendations of previous research (Jovanović and Flanagan, 2014; Padulo et al., 2012; Pareja-Blanco et al., 2016), velocity-based training with a minimum velocity threshold of $80 \%$ maximal attainable velocity would have resulted in a minimum velocity threshold of approximately $0.52 \mathrm{~m} \cdot \mathrm{s}-1$ in the present study. Despite the redistribution of rest during RR4 allowing MV to return to a baseline value after each $52.5 \mathrm{~s}$ rest period, only 28 out of 36 repetitions had a MV greater than $0.52 \mathrm{~m} \cdot \mathrm{s}-1$. Additionally, $120 \mathrm{~s}$ and $30 \mathrm{~s}$ of rest were enough to allow MV to return to baseline during CS4, but only 32 out of 36 repetitions achieved a MV above $0.52 \mathrm{~m} \cdot \mathrm{s}-1$. In RR1, participants were able to complete 36 out of 36 repetitions at a velocity equal to or greater than $0.52 \mathrm{~m} \cdot \mathrm{s}-1$, indicating that RR1 would have allowed for a greater number of repetitions resulting in greater total work and possibly a greater acute training stimulus if minimum velocity thresholds were to be met. Similarly, other researchers have highlighted the effectiveness of inter-repetition rest periods for maintaining velocity (García-Ramos et al., 2015) and suggested that inter-repetition rest periods may be preferential to traditional sets for increasing mechanical stress without decreasing acute performance (Iglesias-Soler et al., 2012, 2013). Although the purpose of this study was not to implement or define velocity-based thresholds, the readers of this manuscript are likely interested in acute kinematics during resistance-training and should consider the aforementioned points when implementing basic cluster sets or rest redistribution protocols during training. In this regard, it appears as though the shorter but more frequent rest intervals used in RR1 may be most beneficial for maintaining movement velocity and power output.

Since the relative external load was the same during each protocol, there was no difference in MF between protocols, in line with previous research (Denton and Cronin, 2006; Moir et al., 2013; Tufano et al., 2016b). There is a lack of
PF data within the cluster set literature, but previously, Hardee et al. (2012) showed that PF was better maintained when longer interrepetition rest periods were used during three cluster sets of six power cleans performed with a load of $80 \%$ of $1 \mathrm{RM}$. On the other hand, Hansen et al. (2011) showed that PF was not different between rest redistribution protocols during four sets of six jump squats with a fixed load of $40 \mathrm{~kg}$. Considering that one study implemented extra rest periods during a heavily loaded concentric movement, whereas the other redistributed the total rest time during a relatively light exercise with a countermovement, it would be difficult to compare the PF results of either of those studies to the data in the present study. However, in a previously published study, PF was reported to be similar between cluster and traditional set protocols inclusive of the same load during the back squat exercise when using different rest period configurations (Tufano et al., 2016b), but a comparison of individual repetitions was lacking, making the present study the first to compare the differences in PF between individual back squat repetitions during cluster sets.

Although PF averaged across 36 repetitions was not different between the current study's protocols, a protocol $\mathrm{x}$ repetition interaction indicates that practical training implications may in fact be present within the data. Specifically, data in Figure 2c show that the repetition that was preceded by a rest period in the CS4 and RR4 protocols displayed less PF than repetitions that were preceded by another repetition. Therefore, PF remained fairly steady during RR1, but was greater during successive repetitions compared to the first repetition of each 4-repetition segment in RR4 and CS4 (Figure 2c). Despite this being the first study to compare PF between individual repetitions of the back squat using cluster sets, the results from a previous study may shed light on this PF phenomenon (Moir et al., 2013). In a study conducted by Moir et al. (2013), participants performed four repetitions of the deadlift in a row (i.e. a traditional set), a cluster set of four individual repetitions with $30 \mathrm{~s}$ of inter-repetition rest, and a cluster set of four repetitions with $30 \mathrm{~s}$ of intra-set rest after every two repetitions. The authors concluded that the additional rest periods during cluster sets had a negative effect on power output and culminated in greater concentric time 
under tension compared to the traditional set (Moir et al., 2013). A lack of peak force data does not allow for a direct comparison with the present study, but an increase in time under tension (and a hypothesized decrease in movement velocity stated by the authors) in the repetitions that followed a $30 \mathrm{~s}$ rest period led the authors to believe that the stretch-shortening cycle was not as profound in the cluster set protocols compared to the traditional set when a repetition was preceded by another repetition (Moir et al., 2013). The authors concluded that the competing mechanisms of fatigue and potentiation resulted in different mechanical responses and that such relationships should always be considered when designing a resistance-training program, especially as inter-set rest periods are employed.

Similar to the protocols used by Moir et al. (2013), the CS4 and RR4 protocols of the present study contained a minimum of $30 \mathrm{~s}$ rest before the repetition that exhibited less PF. Alternatively, the RR1 protocol included only $12 \mathrm{~s}$ of rest between repetitions and did not show the same pattern of decreased PF after a rest period. Therefore, it is possible that there may have been a forcepotentiation mechanism involved that lasted up to $12 \mathrm{~s}$, but not $30 \mathrm{~s}$ during dynamic resistance training with maximal effort in the present study and the study conducted by Moir et al. (2013). In a practical sense, inter-repetition rest periods approaching $30 \mathrm{~s}$ may not result in optimal force production during loaded back squats performed for many repetitions. However, it is important to consider that in addition to PF, other factors such as movement velocity most likely play a larger role for determining acute exercise performance and developing power output (Oliver et al., 2016).
Lastly, previous studies have shown that the redistribution of rest periods maintains the kinetic and kinematic characteristics of resistance training (Hansen et al., 2011; Moreno et al., 2014; Oliver et al., 2016), and the data in the present study also support those findings. Although there were no statistical differences between variables when all 36 repetitions were averaged together within each protocol, it is important that the strength and conditioning professional be cognizant of the competing physiological mechanisms of fatigue and potentiation, and consider the protocol $\mathrm{x}$ repetition interaction patterns of velocity and power output when rest periods are redistributed within a protocol.

The present study demonstrated that redistributing total rest time results in similar overall kinetics and kinematics during barbell back squats in strength-trained men, but resulted in different patterns of force, velocity, and power output throughout the session. Rest periods of 30 $\mathrm{s}$ or greater may dissipate the potential for "priming" the stretch-shortening cycle to produce maximal peak force, which seems to be present when performing up to four successive repetitions, or when performing single repetitions separated by $12 \mathrm{~s}$ of rest. If a minimum velocity threshold must be met, a protocol containing inter-repetition rest periods similar to RR1 may allow for the greatest number of repetitions to be performed. Further research may examine such protocols using different exercises and external loads in addition to determining the effect of various rest redistribution protocols on acute physiological responses that occur during resistance training.

\section{Acknowledgements}

The completion of this study and preparation of this manuscript were partially funded by a postgraduate research scholarship at the Edith Cowan University and a Biological Aspects of Human Movement grant (Progress Q41) from Charles University. Neither the Edith Cowan University nor Charles University influenced the experimental design, data analysis or presentation of the study.

\section{References}

Asadi A, Ramirez-Campillo R. Effects of cluster vs. traditional plyometric training sets on maximal-intensity exercise performance. Medicina, 2016; 52: 41-45

Cormie P, McBride JM, McCaulley GO. Validation of power measurement techniques in dynamic lower body resistance exercises. J Appl Biomech, 2007; 23: 103-118

de Souza TP, Fleck SJ, Simão R, Dubas JP, Pereira B, de Brito Pacheco EM, da Silva AC, de Oliveira PR. Comparison between constant and decreasing rest intervals: influence on maximal strength and 
hypertrophy. J Strength Cond Res, 2010; 24: 1843-1850

Denton J, Cronin JB. Kinematic, kinetic, and blood lactate profiles of continuous and intraset rest loading schemes. J Strength Cond Res, 2006; 20: 528-534

Faigenbaum AD, Kraemer WJ, Blimkie CJR, Jeffreys I, Micheli LJ, Nitka M, Rowland TW. Youth resistance training: updated position statement paper from the national strength and conditioning association. $J$ Strength Cond Res, 2009; 23: S60-S79

Fry AC. The role of resistance exercise intensity on muscle fibre adaptations. Sports Med, 2004; 34: 663-679

García-Ramos A, Padial P, Haff GG, Argüelles-Cienfuegos J, García-Ramos M, Conde-Pipó J, Feriche B. Effect of different inter-repetition rest periods on barbell velocity loss during the ballistic bench press exercise. J Strength Cond Res, 2015; 29: 2388-2396

Girman JC, Jones MT, Matthews TD, Wood RJ. Acute effects of a cluster-set protocol on hormonal, metabolic and performance measures in resistance-trained males. Eur J Sport Sci, 2014; 14: 151-159

Haff GG, Whitley A, McCoy LB, O'Bryant HS, Kilgore JL, Haff EE, Pierce K, Stone MH. Effects of different set configurations on barbell velocity and displacement during a clean pull. J Strength Cond Res, 2003; 17: $95-103$

Hansen KT, Cronin JB, Newton MJ. The effect of cluster loading on force, velocity, and power during ballistic jump squat training. Int J Sports Physiol Perform, 2011; 6: 455-468

Hardee JP, Triplett NT, Utter AC, Zwetsloot KA, McBride JM. Effect of interrepetition rest on power output in the power clean. J Strength Cond Res, 2012; 26: 883-889

Iglesias-Soler E, Boullosa DA, Carballeira E, Sanchez-Otero T, Mayo X, Castro-Gacio X, Dopico X. Effect of set configuration on hemodynamics and cardiac autonomic modulation after high-intensity squat exercise. Clin Physiol Funct Imaging, 2014; 35: 250-257

Iglesias-Soler E, Carballeira E, Sanchez-Otero T, Mayo X, Fernandez-Del-Olmo M. Performance of maximum number of repetitions with cluster set configuration. Int J Sports Physiol Perform, 2013; 9: 637-642

Iglesias-Soler E, Carballeira E, Sanchez-Otero T, Mayo X, Jimenez A, Chapman ML. Acute effects of distribution of rest between repetitions. Int J Sports Med, 2012; 33: 351-358

Jovanović M, Flanagan EP. Researched applications of velocity based strength training. J Aust Strength Cond, 2014; 22: 58-69

Joy JM, Oliver JM, McCleary SA, Lowery RP, Wilson JM. Power output and electromyography activity of the back squat exercise with cluster sets. J Sports Science, 2013; 1: 37-45

Lawton TW, Cronin JB, Lindsell RP. Effect of interrepetition rest intervals on weight training repetition power output. J Strength Cond Research, 2006; 20: 172-176

Matuszak ME, Fry AC, Weiss LW, Ireland TR, McKnight MM. Effect of rest interval length on repeated 1 repetition maximum back squats. J Strength Cond Res, 2003; 17: 634-637

Medeiros HS, Mello RS, Amorim MZ, Koch AJ, Machado M. Planned intensity reduction to maintain repetitions within recommended hypertrophy range. Int J Sports Physiol Perform, 2013; 8: 384-390

Moir GL, Graham BW, Davis SE, Guers JJ, Witmer CA. Effect of cluster set configurations on mechanical variables during the deadlift exercise. J Hum Kinet, 2013; 39: 15-23

Moreno SD, Brown LE, Coburn JW, Judelson DA. Effect of cluster sets on plyometric jump power. J Strength Cond Res, 2014; 28: 2424-2428

Myer GD, Ford KR, Palumbo OP, Hewett TE. Neuromuscular training improves performance and lowerextremity biomechanics in female athletes. J Strength Cond Res, 2005; 19: 51-60

Oliver JM, Kreutzer A, Jenke S, Phillips MD, Mitchell, JB, Jones MT. Acute response to cluster sets in trained and untrained men. Eur J Appl Physiol, 2015; 115: 2383-2393 
Oliver JM, Kreutzer A, Jenke SC, Phillips MD, Mitchell JB, Jones MT. Velocity drives greater power observed during back squat using cluster sets. J Strength Cond Res, 2016; 30: 235-243

Padulo J, Mignogna P, Mignardi S, Tonni F, D'Ottavio S. Effect of different pushing speeds on bench press. Int J Sports Med, 2012; 33: 376-380

Pareja-Blanco F, Rodriguez-Rosell D, Sanchez-Medina L, Sanchis-Moysi J, Dorado C, Mora-Custodio R, Yanez-Garcia JM, Morales-Alamo D, Perez-Suarez I, Calbet JA, Gonzalez-Badillo JJ. Effects of velocity loss during resistance training on athletic performance, strength gains and muscle adaptations. Scand J Med Sci Sports, 2016 (epub ahead of print) doi: 10.1111/sms.12678

Selye H. Stress and the general adaptation syndrome. Br Medical J, 1950; 1: 1383

Tufano JJ, Brown LE, Haff GG. Theoretical and practical aspects of different cluster set structures: a systematic review. J Strength Cond Res, 2017; 3: 848-867

Tufano JJ, Conlon JA, Nimphius S, Brown LE, Banyard HG, Williamson BD, Bishop LG, Hopper AJ, Haff GG. Cluster sets permit greater mechanical stress without decreasing relative velocity. Int J Sports Physiol Perform, 2016a (epub ahead of print).

Tufano JJ, Conlon JA, Nimphius S, Brown LE, Seitz LB, Williamson BD, Haff GG. Cluster sets maintain velocity and power during high-volume back squats. Int J Sports Physiol Perform, 2016b; 7: 885-892

\section{Corresponding author:}

\section{James Tufano}

Charles University, Faculty of Physical Education and Sport

31 Jose Martiho, Prague, Czech Republic, 16252

James.J.Tufano@gmail.com

+420777144962 